\title{
Power load forecasting algorithm based on nonlinear inertial factor change pattern particle swarm optimization algorithm
}

\author{
Jin Liang ${ }^{1}$, Wang Yongzhi ${ }^{1, *}$, and Bao Xiaodong ${ }^{1}$ \\ ${ }^{1}$ College of Instrumentation \& Electrical Engineering, Jilin University, Changchun, China
}

\begin{abstract}
The common method of power load forecasting is the least squares support vector machine, but this method is very dependent on the selection of parameters. Particle swarm optimization algorithm is an algorithm suitable for optimizing the selection of support vector parameters, but it is easy to fall into the local optimum. In this paper, we propose a new particle swarm optimization algorithm, it uses non-linear inertial factor change that is used to optimize the algorithm least squares support vector machine to avoid falling into the local optimum. It aims to make the prediction accuracy of the algorithm reach the highest. The experimental results show this method is correct and effective.
\end{abstract}

\section{Introduction}

In the construction of power system, it not only needs to classify the electric energy in a safe and scientific manner, but also needs to meet the user load requirements at any time. Because the power system's ability to store electrical energy is not very powerful, accurate power load forecasting can greatly improve the efficiency of the use of electrical energy and effectively avoid the waste of electrical energy[1]. In addition, the power load forecasting has great help to grid point control and real-time power system dispatch. At present, the algorithms based on Least squares support vector machine (LS-SVM) are widely used in short-term power load forecasting. Particle swarm optimization of this paper as an optimization means, the design of particle swarm optimization LSSVM algorithm. At the same time, the non-linear change of inertia factor is introduced to improve the prediction accuracy of the algorithm.

\section{Least square support vector machines}

Support Vector Machine (SVM) is a machine learning method based on statistical VC dimension theory. The basic idea of SVM is to find a classification line or classification hyperplane that can correct the data and minimize the interval[2]. SVM has the advantages of simple structure, strong promotion ability, fast learning speed and easy convergence when solving. Converting SVM classification problem to nearest SVR, and figure 1 shows the basic principle[3].

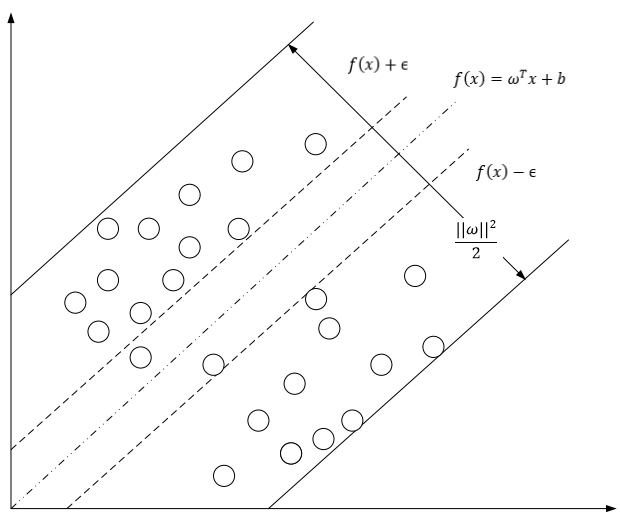

Fig. 1. Principle diagram of Support vector regression.

Least squares support vector machine is an extension of the standard SVM, with fewer parameters to choose than traditional support vector machines. It replaces the inequality constraint with an equation, and replaces the original loss function with the sum of the squared errors, which makes the original computational task simplified from the quadratic programming to solving the linear equations, making the model easier to solve[4]

It is assumed that the nonlinear load forecasting model is:

$$
f(x)=\left(\omega^{T} \varphi(x)\right)+b
$$

Based on the concept of LS-SVM, the original structure minimization model as shown in Fig. 1 can be expressed as (2), and its constraint is converted from inequality to equality.

\footnotetext{
* Corresponding author: iamwangyongzhi@126.com
} 


$$
\begin{aligned}
& \min \frac{1}{2}\|\omega\|^{2}+\frac{1}{2} \gamma \sum_{i=1}^{n} e_{i}^{2} \\
& \text { s.t. } \mathrm{f}(\mathrm{x})+\mathrm{e}_{i}=y_{i}, i=1,2 \ldots n
\end{aligned}
$$

In (2): $e_{i}$ is an error, belongs to $\mathrm{n} \times 1$ vector, $y$ is a regularization parameter, $f(x)$ is the objective function.

The introduction of Lagrange multiplier $\lambda$ formula (2) can be transformed into:

$$
\begin{aligned}
& \min \frac{1}{2}\|\omega\|^{2}+\frac{1}{2} \gamma \sum_{i=1}^{n} e_{i}^{2}- \\
& -\sum_{i=1}^{n} \lambda_{i}\left(\omega^{T} \varphi\left(x_{i}\right)+b+\mathrm{e}_{i}-y_{i}\right)
\end{aligned}
$$

From the KKT conditions, the various equations are deduced, and the linear equations shown in (4) can be obtained:

$$
\left|\begin{array}{cc}
0 & \overrightarrow{1}_{n}^{T} \\
\overrightarrow{1}_{n} & K_{i j}+\frac{l_{n}}{\gamma}
\end{array}\right|\left|\begin{array}{l}
b \\
\vec{\lambda}
\end{array}\right|=\left|\begin{array}{c}
0 \\
\vec{y}
\end{array}\right|
$$

Further into

$$
\mathrm{y}=\sum_{i=1, j=1}^{n} \lambda_{i} K\left(x_{\mathrm{i}}, x_{j}\right)+b
$$

In (5): $b$ is the displacement term, $\lambda$ is Lagrange multiplier, $l n$ is $\mathrm{n} \times \mathrm{n}$ unit vector, $1 n$ is $\mathrm{n} \times 1$ dimensional column vector, Kij is kernel function.

In this way, the regression equation of power load forecasting is obtained, while the introduction of kernel function simplifies the calculation, and the forecasting power load data can be obtained by using the training data to calculate the required value and bring it into the predictor variables.

LS-SVM needs to adjust parameters to improve accuracy. How to determine the current parameter as the optimal parameter is a thorny issue. Therefore, the particle swarm optimization algorithm was introduced to optimize the LS-SVM so that the selected parameters were optimal. The comparison of the results before and after the optimization will be shown in Figure 2 in Section 5.

\section{Particle swarm optimization}

Particle swarm optimization (PSO) is an evolutionary algorithm based on iterative optimization design. The algorithm iterates over random particle swarms, and each iteration records two values of the particle (the optimal extremum $P i$ and the global optimum extremum $P g$ ). According to these two extremes, the particle velocity and position are continuously adjusted until the optimal optimization result is obtained[5]. Assuming that the search space is ddimensional and the number of particles is $\mathrm{m}$, then the ith particle can be abstracted as a d-dimensional vector and its position in space can be represented by $x i d$.

$$
\mathrm{x}_{i d}=\left(x_{i 1}, x_{i 2}, \ldots x_{i d}\right), i=1,2 \ldots m
$$

Similarly, the velocity of the particle is also expressed as a vector of the same dimension Vid expressed as:

$$
\mathrm{V}_{i d}=\left(V_{i 1}, V_{i 2}, \ldots V_{i d}\right), i=1,2 \ldots m
$$

And record each optimal position $P$ id:

$$
\mathrm{P}_{i d}=\left(P_{i 1}, P_{i 2}, \ldots P_{i d}\right), i=1,2 \ldots m
$$

And search only the population of the global optimum position $P_{i g}$ :

$$
\mathrm{P}_{\mathrm{i} g}=\left(P_{i 1}, P_{i 2}, \ldots P_{i d}\right), i=1,2 \ldots m
$$

The algorithm speed and location update formula we use is:

$$
\begin{aligned}
& V_{\mathrm{id}}^{k+1}=\omega_{g} V_{\mathrm{id}}^{k}+C_{1} \alpha\left(P_{i d}^{k}-x_{i d}^{k}\right) \\
& +C_{2} \beta\left(P_{g d}^{k}-x_{i d}^{k}\right) \\
& x_{i d}=x_{i d}+V_{i d}
\end{aligned}
$$

In (10): $\omega_{g}$ is the inertia factor, $C_{1}, C_{2}$ are acceleration constants, non-negative, $\alpha, \beta$ are 0 to 1 random numbers.

In particle swarm optimization, the selection of inertia factor and the choice of acceleration constant determine whether the particle swarm will fall into local optimum, the convergence speed of the algorithm and whether there will be oscillation phenomenon[6-8]. This paper selects the nonlinear inertia factor change method to optimize Particle swarm algorithm.

Select non-linear inertia factor update method:

$$
\omega_{\mathrm{g}}=\omega_{\min }\left(\omega_{\max } / \omega_{\min }\right)^{1 /(1+10 \text { Count } / \text { LoopC })}
$$

In(11): $\omega_{\max }, \omega_{\min }$ respectively maximum and minimum inertia factor, Count and LoopC the current iteration number and the iteration upper limit.

This paper uses this method to optimize particle swarm parameters to improve the overall algorithm accuracy.

\section{Pso-based Is-svm algorithm}

\subsection{Data preprocessing}

The obtained power load data are processed and the data are classified[9].The power load forecasting data can be divided into three categories:

Typical load component: the main component of the power load data, the composition of different types of load and its share of these loads with periodic changes and linear changes in the characteristics and 
have different basic load characteristics, the corresponding response of different factors.

Weather-sensitive components: The power load is more affected by weather factors, and the component of this part mainly selects temperature and humidity data[10].

Abnormal or special event load components: The load will be greatly affected by the system failure, power cuts, special festivals, natural disasters and other special events and anomalous events.

\subsection{Random load component processing}

The load data contains hard to explain or unexplained random load components, select the appropriate model and algorithm processing. Because of the magnitude and dimension of the data are not the same, in order to avoid the magnitude of a certain data affect the entire prediction index, the data to do the normalization. For logarithm of power load data, the date is converted to a decimal between $[0,1]$. The difference between temperature and humidity minus the minimum of the current day and divided by the maximum and minimum of the current day is converted into a decimal of the same interval.

\section{Simulation prediction results}

In order to verify the prediction effect of the support vector machine algorithm model based on least squares, a city's real power load data is used for verification, and the error uses the RMS relative error. To avoid the influence of single data, multi-groups of multi-day data are selected in the test data. LS-SVM in Table 1 is an unoptimized prediction model, WOPso is a particle swarm optimization algorithm prediction model using conventional linear fixed inertia factor, W1Pso refers to a particle swarm optimization algorithm prediction model that uses an adaptive linear inertia factor, and W2Pso is a particle swarm optimization algorithm prediction model that uses an adaptive nonlinear inertia factor. table 1 shows the results of model verification and prediction.

Table 1. Prediction results of various methods

\begin{tabular}{|c|c|c|c|c|}
\hline Data & LS-SVM & W0Pso & W1Pso & W2Pso \\
\hline $3 / 17$ & $3.48 \%$ & $1.72 \%$ & $1.60 \%$ & $1.60 \%$ \\
\hline $3 / 18$ & $2.44 \%$ & $1.80 \%$ & $1.75 \%$ & $1.71 \%$ \\
\hline $3 / 19$ & $2.32 \%$ & $1.67 \%$ & $1.56 \%$ & $1.54 \%$ \\
\hline
\end{tabular}

\begin{tabular}{|c|c|c|c|c|}
\hline $3 / 20$ & $2.73 \%$ & $1.81 \%$ & $1.44 \%$ & $1.43 \%$ \\
\hline $3 / 21$ & $1.72 \%$ & $1.68 \%$ & $1.68 \%$ & $1.45 \%$ \\
\hline $3 / 22$ & $3.28 \%$ & $2.60 \%$ & $2.30 \%$ & $2.30 \%$ \\
\hline $3 / 23$ & $4.48 \%$ & $1.47 \%$ & $1.44 \%$ & $1.41 \%$ \\
\hline $3 / 24$ & $2.03 \%$ & $1.27 \%$ & $1.27 \%$ & $1.26 \%$ \\
\hline $3 / 25$ & $3.20 \%$ & $2.27 \%$ & $2.25 \%$ & $2.24 \%$ \\
\hline
\end{tabular}

It can be seen that no matter how the LS-SVM performance is improved, all the algorithms improve the prediction accuracy obviously. The adaptive particle swarm optimization algorithm that uses nonlinear inertial factor change can achieve a stable prediction accuracy and the highest prediction accuracy, and the average prediction accuracy can reach about $2 \%$, which can meet the real needs. According to the British studies, the annual cost savings of 17.7 million yuan can be saved for every $1 \%$ increase in the short-term power load accuracy[11-12]. The optimization of PSO with nonlinear inertia factor is almost all better than that with linearly varying particle swarm optimization Algorithm, so using nonlinear change algorithm model can provide more accurate power system budget, resulting in significant cost savings. Figure 3 uses the line graph to compare the results of multiple algorithms to give an intuitive comparison, we can see that the optimized prediction curve and the original data curve fitting better.

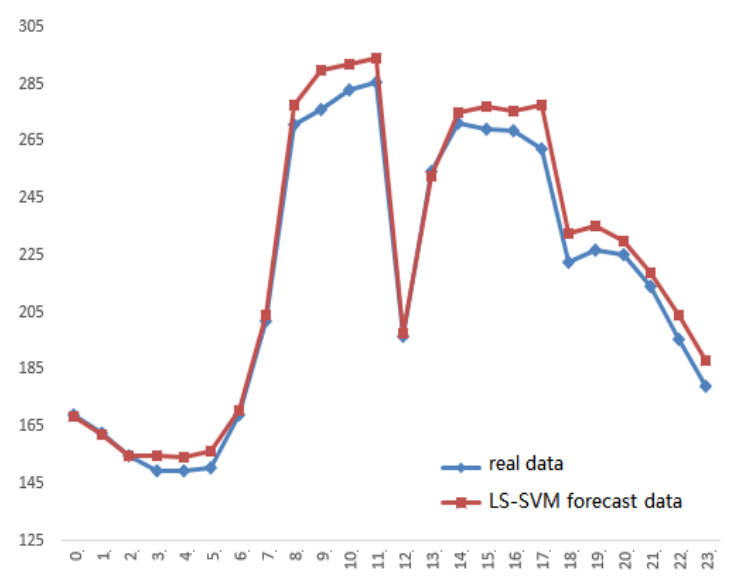

Fig. 2. Unoptimized LS-SVM Prediction Results. 


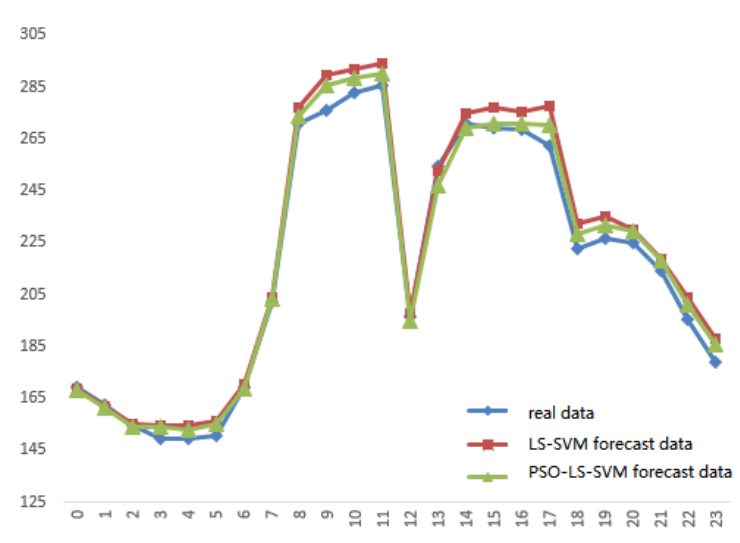

Fig. 3.Comparison of prediction results.

\section{CONCLUSION}

In this paper, a variety of inertia factor change methods and adaptive particle swarm optimization LSSVM power forecasting model are used to predict power load, and the following conclusions are drawn: The prediction results of non-linear inertia factor change are generally superior to those of linear variation prediction results.

It is found that the forecasting model of power load using adaptive particle swarm optimization with nonlinear inertia factor change has the highest prediction accuracy of about $2 \%$ when the fluctuation of power load data does not appear violent fluctuation. The proof algorithm can meet the daily scheduling requirements of the power system, and the algorithm has availability and practicality. When verifying the algorithm, for some time points where the data fluctuates, the prediction accuracy is difficult to guarantee, and may be related to special days or unusual weather. There is no shortage of data with very high prediction accuracy in the test results, which may result from "over-learning" due to limited data sets. Follow-up will increase the test data to further verify and improve the algorithm

\section{Acknowledgments}

This research has been jointly supported by National Key R \& D Program of China (2016YFC0600501), National Natural Science Foundation of China (2016YFC0600501-4) and the State Key Program of National Natural Science of China(41430320).

\section{References}

1. Niu Dongxiao, Cao Shuhua, Zhao Lei, et al. Power load forecasting technology and its application. (Beijing: Chinese Power Press,1998)
2. Zhang Xiaojun, Lily Tien, Feng Zhimin, Wu Daolin, et al. Journal of Jingchu Institute of technology,30,48-52(2015)

3. Yongzhi Wang, Yuli Zhang, Jining Yi, et al., Mathematical Problems In Engineering(2014).

4. Li Xiao,Wang Xin,Zheng Yihui,et.al.,.Power System Protection and Control, 43,1-11(2015)

5. Dong Yong, Guo Haimin.;Adaptive chaos particle swarm optimization based on colony fitness variance. Application research of Computers, 28 , 854-856 (2011)

6. Chen Guimin, Jia Jianyuan,Han Qi. Journal of Xi'an Jiao Tong University, 40,53-56 (2006)

7. Feng hao, Li Xianwei.;.Journal of Bengbu University, 4,21-24(2015).

8. Ma Xiaojin, Zhu Bo, Dai Lin, et al.; Control Theory and Applications, 35 (2016)

9. Liu Jia, Li Dan, Gao Liqun, Lu Shun.;Journal of Northeastern University (Natural Science),09, 12291232 (2007)

10. Wang Yijun, Li Dianwen, Gao Electrical Measurement \& Instrument, 52 , 22-25 (2015)

11. Sun Caixin, Zhang Xiaoxing, Cheng Qiyun, et.al. Transactions of China Electrotechnical Society, 10,53-58(2004)

12. Yongzhi Wang,Yuli Zhang,Fuliang Zhang,et al.. Mathematical Problems In Engineering,(2013) 